ORIGINALES

\title{
Estudio empírico de las variables de la Teoría de la Conducta Planificada como factores de riesgo para el consumo de cocaína en tres grupos diferentes
}

\section{Empirical study of the Planned Behavior Theory variables as risk factors for cocaine use in three different groups}

| Jesús SAIz GaLdós
Departamento de Psicología Social, Facultad de Ciencias Políticas y Sociología, Universidad Complutense de Madrid

Enviar correspondencia a:

Jesús Saiz, Despacho 1613, Departamento de Psicología

Social, Facultad de Ciencias Políticas y Sociología, Universidad

Complutense de Madrid, Campus de Somosaguas, 28223 Madrid.

E-mail: jesus_saiz@hotmail.com.

\section{RESUMEN}

Objetivo: Explorar empíricamente la presencia de determinados factores como elementos de riesgo para el consumo de cocaina.

Método: Estudio transversal que compara las puntuaciones obtenidas en un conjunto de items que miden las variables de la Teoria de la Conducta Planificada (actitud, norma subjetiva, control conductual percibido e intención conductual) en tres grupos: el grupo A está formado por 192 sujetos consumidores de cocaina sin tratamiento, el grupo B está formado por 110 sujetos consumidores de cocaina en tratamiento y el grupo $\mathrm{C}$ lo componen 100 sujetos que nunca habian consumido cocaina. Resultados: El grupo A puntuó significativamente más alto en todas las variables, mientras que el grupo $C$ fue el que obtuvo menores puntuaciones. El grupo B no mostró diferencias significativas con el grupo $A$ en la variable norma subjetiva, ni con el grupo $C$ en las variables control conductual percibido e intención conductual.

Discusión: Se subraya la importancia de las actitudes y la norma subjetiva para la explicación del consumo actual de cocaína, pero especialmente, la función del control conductual percibido como factor de riesgo necesario a tomar en cuenta en el diseño de medidas preventivas.

Palabras clave: Cocaina, actitudes, control conductual percibido, norma subjetiva, estudio descriptivo.

\section{ABSTRACT}

Objective: To explore empirically the presence of certain factors as risk elements for cocaine consumption.

Method: Analysis of the data obtained in a set of items that measure the variables of the Planned Behavior Theory (attitude, subjective norm, perceived behavioral control and behavioral intention) in three groups: Group A is made up of 192 cocaine users without treatment, Group B comprises 110 cocaine users in treatment, and Group $C$ is made up of 100 individuals who had never used cocaine.

Results: Group A scored significantly higher in all the variables, while Group C was which obtained the lowest scores. Group B showed no significant differences from Group A in the subjective norm variable, nor from Group $C$ in the perceived behavioral control and behavioral intention variables.

Discussion: This study highlights the importance of attitudes and subjective norm in the explanation of current cocaine use, but especially the function of perceived behavioral control as a risk factor that should be taken into account in the design of preventive strategies.

Key words: Cocaine, attitudes, perceived behavioral control, subjective norm, descriptive study. 


\section{INTRODUCCIÓN}

La cocaina es una droga estimulante cuyo consumo ha aumentado en los últimos años (OED, 2005), siendo España, de hecho, el país que mayor porcentaje de consumo de cocaína demuestra en todo el mundo (UNODC, 2007). Además, diversos autores (Calafat, Juan, Becoña, Fernández, Gil, Palmer, et al., 2000) han encontrado que la cocaina es la tercera droga "de moda" en España, sólo por detrás del alcohol y el cannabis, estando la cocaína muy vinculada a la diversión por sus efectos estimulantes. Así, el incremento en los casos de sujetos que solicitan tratamiento para dejar de consumir esta droga, junto con el número de accidentes, muertes y problemas relacionados con la misma no dejan de sorprender a las autoridades que buscan la manera de atajarlo (Estrategia Nacional sobre Drogas, 2000-2008). En este sentido, un primer paso obligado para la elaboración de campañas preventivas es conocer los perfiles psicosociales de los consumidores. En este ámbito existe una importante variedad de estudios que exploran la presencia de variables tanto individuales (Pedrero, 2003; Sáiz, González, Paredes, Martínez y Delgado, 2001) como sociales (Navarro, 2000; Saiz, 2007). Aquí se hace uso de la Teoría de la Conducta Planificada para describir algunos elementos psicosociales que podrian determinar el consumo de cocaína.

\section{Aproximación teórica: la Teoría de la Conducta Planificada}

Ajzen y Fishbein $(1980)$ y Ajzen $(1988,1991)$ consideraron que la mejor manera de comprender y predecir las conductas de los sujetos es conociendo sus intenciones a actuar. Así, elaboraron una primera teoría que hacía énfasis en la relación entre Actitud, Norma subjetiva e Intención conductual a la que denominaron Teoría de la Acción Razonada (TAR) (Ajzen y Fishbein, 1980). Sin embargo, revisiones posteriores señalaron que algunas conductas no estaban bajo el control completo del individuo y requerían conductas cooperativas, por lo que Ajzen $(1988,1991)$ propuso la Teoría de la Conducta Planificada (TCP), que amplía la anterior introduciendo el concepto de Control conductual percibido

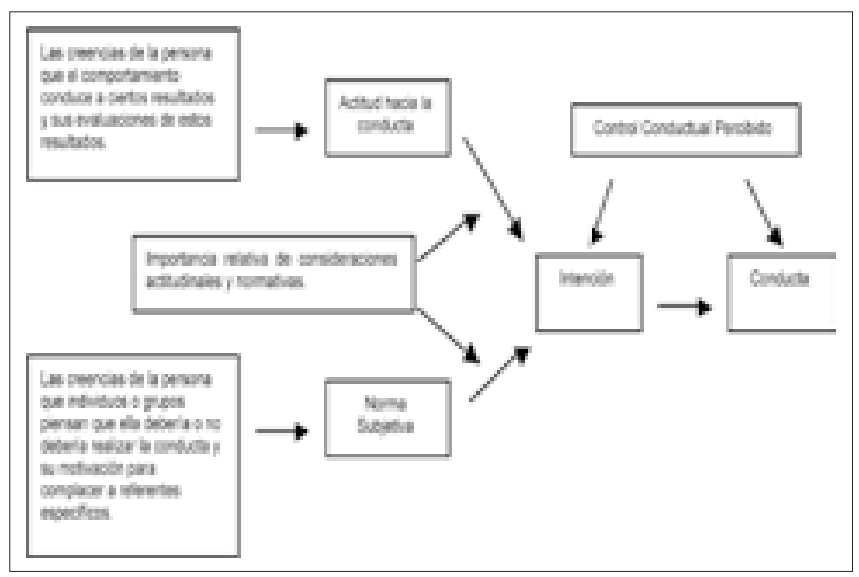

Figura 1. Diagrama de la Teoría de la Conducta Planificada (Ajzen, 1991).
Tal y como se muestra en la figura 1, para estos autores (Ajzen y Fishbein, 1980; Schifter y Ajzen, 1985): 1) La Intención conductual es el factor más directo y cercano a la conducta. 2) La Actitud es una valoración positiva o negativa que el sujeto hace de la realización de la conducta, y está determinada por las creencias de la persona sobre los resultados del comportamiento y sus evaluaciones de estos resultados. 3) La Norma subjetiva consiste en la percepción que el sujeto tiene de las presiones sociales a que realice $u$ omita cierta conducta, y está compuesta por las creencias de la persona sobre lo que determinados grupos de referencia piensan de dicho comportamiento y su motivación para complacer a estos grupos de referencia. Finalmente, 4) el Control conductual percibido representa la percepción de la facilidad o dificultad para realizar una conducta, y se asume que refleja la experiencia pasada así como la anticipación de impedimentos y obstáculos.

\section{Soporte empírico de la Teoría de la Conducta Planificada aplicada al consumo de sustancias}

Desde este marco teórico (TAR y TCP), se han realizado importantes investigaciones que han permitido comprender mejor los factores determinantes del consumo de sustancias como el cannabis (Conner y McMillan, 1999; Humphrey, O'Malley, Johnston y Bachman, 1988; 0'Callaghan y Hannon, 2003; Olivar y Carrero, 2007), el alcohol (McMillan y Conner, 2003; Norman y Conner, 2006; Trujillo, Forns y Pérez, 2007), el tabaco (Conner, Sandberg, McMillan y Higgins, 2006; Côté, Godin y Gagné, 2004; McMillan y Conner, 2003; Synnøve y Rise, 2006), las bezodiacepinas (Hulten, Bakker, Lodder, Teeuw, Bakker y Leufkens, 2003), el MDMA (Umeh y Patel, 2004) y la cocaina (Levy y Pierce, 1989).

Asi por ejemplo, Humphrey et al. (1988) encontraron, en una muestra de 27.842 jóvenes estadounidenses, que el consumo de marihuana estaba influido por las actitudes hacia el mismo $(r=0,57, p<0,01)$ y la norma subjetiva, verificando una importante correlación entre las actitudes, la norma subjetiva y el consumo de marihuana en el último mes. La norma subjetiva estaba compuesta por la influencia de los amigos $(r=0,53, p<0,01)$, la religión $(r=-0,24, p<0,01)$ y la escuela $(r=-0,21, p<0,01)$.

En relación con el consumo de tabaco y alcohol, entre los distintos estudios que han empleado este marco teórico, el de McMillan y Conner (2003) muestra la capacidad predictiva, especialmente de las actitudes y del control conductual percibido, tanto en la intención de consumo (alcohol $\mathrm{R}^{2}=$ 0.167 ; tabaco $R^{2}=0.137$ ), como en el consumo real realizado a los seis meses de seguimiento (alcohol $R^{2}=0.289$; tabaco $\mathrm{R}^{2}=0.423$ ).

Por otra parte, Hulten et al. (2003) compararon la influencia de las variables de la TCP en dos grupos de sujetos que hacian uso de Benzodiacepinas (BZP), el primero estaba formado por 360 sujetos con experiencia previa de BZP y el segundo por 107 sujetos sin experiencia con BZP, encontrando que, con un rango de respuesta $1-5$, quienes no tenían experiencia previa, mostraron un mayor control con- 
ductual $(4,40$ vs. 3,$65 ; p<0,001)$ y estaban más influenciados por la norma subjetiva $(3,61$ vs, 3,$31 ; p<0,01)$. Mientras que aquellos que tenían una historia más extensa de uso de BZP, tenian mayor intención conductual $(4,50$ vs. 3,$41 ; p<0,001)$ y actitudes favorables $(3,64$ vs. 3,$36 ; p<0,001)$. Además, los autores hallaron que la TCP explicaba el $67 \%$ de la varianza de la conducta de consumir BZP para los consumidores experimentados, aunque sólo el 18\% para los inexpertos.

Los factores que incluye la TCP también han sido contrastados en estudios que exploran el consumo de MDMA. De esta manera, Umeh y Patel (2004), con una muestra de 200 estudiantes universitarios, encontraron que la interacción entre la conducta pasada, las actitudes y la norma subjetiva predecía un $64,2 \%$ de la varianza, mientras que la conducta pasada, las actitudes y el control conductual predecian un 69,2\% de la varianza.

Finalmente, en el estudio llevado a cabo por Levy y Pierce (1989) con una muestra de 1.002 jóvenes australianos, se pudo observar que dentro de los distintos grupos de edad explorados, cuantas más actitudes a favor del consumo de cocaína poseían los sujetos, existía mayor intención de consumirla. Además, cuando el sujeto conocía a alguien que consumiese cocaína en su círculo social, éste tenía más intención de consumirla que si no conociese a nadie.

De esta manera, la TCP se ha mostrado en anteriores investigaciones como un marco referencial teórico comprensivo, que permite describir con éxito algunos de los factores psicosociales que mejor explican el consumo de sustancias. Dada la urgente necesidad que tenemos de proveer de descripciones detalladas, que permitan reconocer aquellos elementos que habrán de emplearse en las distintas campañas preventivas, a continuación se describe un estudio que tiene por objetivo general explorar las variables de la TCP en consumidores de cocaína, sujetos en tratamiento por dependencia a esta sustancia y sujetos que nunca la han consumido. En líneas generales, tomando en cuenta los resultados de los estudios comentados, se esperaría que, sujetos consumidores de cocaína mostrasen una actitud más favorable al consumo de la misma, una mayor norma subjetiva, un control conductual más elevado y una mayor intención de consumir cocaína que aquellos que están en tratamiento por dependencia a esta sustancia o que quienes nunca la han probado.

\section{MATERIAL Y MÉTODOS}

\section{Participantes y procedimiento}

El siguiente estudio reúne una muestra de 402 sujetos, los cuales han sido divididos en tres grupos, tomando en cuenta si declaraban consumir cocaína ocasionalmente (grupo A), estar en tratamiento por dependencia de la misma (grupo B) o nunca haberla consumido (grupo C). Las características sociodemográficas y de consumo se detallan en la tabla 1.
Tabla 1. Características sociodemográficas y de consumo de los grupos comparados

\begin{tabular}{|c|c|c|c|c|}
\hline & & Grupo A & Grupo B & Grupo C \\
\hline \multirow[t]{5}{*}{ Rango de edad } & $20-24$ años ( = 1) & $56,3 \%$ & $37,3 \%$ & $49,0 \%$ \\
\hline & $25-29$ años ( = 2) & $30,2 \%$ & $48,2 \%$ & $43,0 \%$ \\
\hline & 30-34 años ( = 3) & $9,4 \%$ & $10,0 \%$ & $5,0 \%$ \\
\hline & 35-39 años ( = 4) & $2,1 \%$ & $3,6 \%$ & $3,0 \%$ \\
\hline & 40-44 años ( = 5) & $2,1 \%$ & $0,9 \%$ & $0,0 \%$ \\
\hline \multicolumn{2}{|l|}{ Total } & $100 \%$ & $100 \%$ & $100 \%$ \\
\hline \multicolumn{2}{|l|}{ Media } & 1,64 & 1,83 & 1,62 \\
\hline \multicolumn{2}{|l|}{ Desv. Típica } & 0,893 & 0,822 & 0,722 \\
\hline \multirow[t]{2}{*}{ Género } & Varón (=1) & $63,5 \%$ & $72,7 \%$ & $70,0 \%$ \\
\hline & Mujer ( = 2) & $36,5 \%$ & $27,3 \%$ & $30,0 \%$ \\
\hline \multicolumn{2}{|l|}{ Total } & $100 \%$ & $100 \%$ & $100 \%$ \\
\hline \multicolumn{2}{|l|}{ Media } & 1,36 & 1,27 & 1,30 \\
\hline \multicolumn{2}{|l|}{ Desv. Típica } & 0,483 & 0,447 & 0,461 \\
\hline Edad de inicio & 15-19 años ( = 1) & $53,9 \%$ & $61,7 \%$ & \\
\hline de consumo de & $20-24$ años ( = 2) & $35,6 \%$ & $26,2 \%$ & \\
\hline \multirow[t]{3}{*}{ Cocaina } & $25-29$ años ( = 3) & $7,3 \%$ & $9,3 \%$ & \\
\hline & 30-34 años ( = 4) & $2,6 \%$ & $1,9 \%$ & \\
\hline & 35-39 años ( = 5) & $0,5 \%$ & $0,9 \%$ & \\
\hline \multicolumn{2}{|l|}{ Total } & $100 \%$ & $100 \%$ & \\
\hline \multicolumn{2}{|l|}{ Media } & 1,60 & 1,54 & \\
\hline \multicolumn{2}{|l|}{ Desv. Típica } & 0,781 & 0,816 & \\
\hline \multirow{5}{*}{$\begin{array}{l}\text { Cada cuánto } \\
\text { consume } \\
\text { Cocaina }\end{array}$} & Una vez al mes o menos ( = 1) & $65,6 \%$ & $6,7 \%$ & \\
\hline & Dos o tres veces al mes ( = 2) & $18,5 \%$ & $9,6 \%$ & \\
\hline & Todos los fines de semana $(=3)$ & $11,1 \%$ & $19,2 \%$ & \\
\hline & Varias veces durante la semana $(=4)$ & $4,2 \%$ & $37,5 \%$ & \\
\hline & Todos los dias $(=5)$ & $0,5 \%$ & $26,9 \%$ & \\
\hline \multicolumn{2}{|l|}{ Total } & $100 \%$ & $100 \%$ & \\
\hline \multicolumn{2}{|l|}{ Media * } & $1,56^{*}$ & $3,68^{*}$ & \\
\hline \multicolumn{2}{|l|}{ Desv. Típica } & 0,889 & 1,168 & \\
\hline \multirow{6}{*}{$\begin{array}{l}\text { Tiempo que } \\
\text { Ileva en } \\
\text { tratamiento }\end{array}$} & No estoy en tratamiento ( = 1) & $100,0 \%$ & & \\
\hline & Menos de un mes ( = 2) & & $15,4 \%$ & \\
\hline & Entre uno y tres meses $(=3)$ & & $29,7 \%$ & \\
\hline & Entre tres y seis meses ( = 4) & & $27,5 \%$ & \\
\hline & Entre seis meses y un año ( = 5) & & $6,6 \%$ & \\
\hline & Más de un año ( = 6) & & $20,9 \%$ & \\
\hline \multicolumn{2}{|l|}{ Total } & & $100 \%$ & \\
\hline \multicolumn{2}{|l|}{ Media * } & $1,00^{*}$ & $3,88^{*}$ & \\
\hline \multicolumn{2}{|l|}{ Desv. Típica } & 0,00 & 1,349 & \\
\hline \multicolumn{2}{|l|}{$\mathrm{N}$} & 192 & 110 & 100 \\
\hline \multicolumn{5}{|l|}{${ }^{*} p<0,05$} \\
\hline
\end{tabular}

El grupo de consumidores de cocaína está formado por 192 sujetos residentes en la Comunidad de Madrid. El criterio de inclusión fue que reconociesen consumir cocaína con cierta regularidad (desde "una vez al mes o menos" hasta "todos los días"), sola o asociada a otras sustancias con excepción de la heroína. El intervalo de edad más frecuente se sitúa entre los 20 y 29 años (86,5\%), siendo la mayoría varones $(63,5 \%)$. La edad de inicio de consumo principal es entre los 15-19 años $(53,9 \%)$ y la mayor frecuencia de consumo es de "una vez al mes o menos" (65,6\%), lo cual por otra parte, justificaría el que puedan ser considerados consumidores ocasionales o no problemáticos de una sustancia con gran poder adictivo. Este grupo se reunió por "contactos en cadena", en donde a partir de un consumidor se accede por referencias a otros. Además, se solicitó participantes para el estudio en la prensa (20 Minutos de Madrid y Gaceta Universitaria de la UCM) y mediante la colocación de carte- 
les y distribución de volantes en zonas estratégicas (alrededor de 2.000 carteles y 300 volantes).

El grupo de consumidores de cocaína en tratamiento lo componen 110 sujetos residentes en la Comunidad de Madrid. El principal criterio de inclusión fue que se encontrasen en tratamiento por dependencia a la cocaína, sola o asociada a otras sustancias, con excepción de la heroína. El intervalo de edad más frecuente es entre los 20 y 29 años (85,5\%), siendo la mayoría varones $(72,7 \%)$. La edad de inicio de consumo se localiza entre los 15-19 años (61,7\%), la mayor frecuencia de consumo se localiza en el item "varias veces durante la semana" (37,5\%), mientras que el tiempo que llevan en tratamiento varía desde "menos de un mes" $(15,4 \%)$, hasta "más de un año" (20,9\%). Este grupo está formado por sujetos que acudian a distintos Centros de Atención Integral a Drogadictos (CAID) y a otros centros para la rehabilitación de la Comunidad de Madrid (públicos y privados)'.

Finalmente, el grupo de no consumidores está formado por 100 sujetos, estudiantes de distintas carreras universitarias, a los cuales les fueron aplicados los cuestionarios en diferentes instituciones de enseñanza de la Comunidad de Madrid (públicas y privadas) ${ }^{2}$. El criterio de inclusión es que no hubiesen consumido nunca cocaína. El intervalo de edad más frecuente se encuentra entre los 20 y 29 años (92\%), siendo la mayoría varones (70\%).

Cuando fue viable la aplicación de los cuestionarios se realizó de manera colectiva. Así se procedió en aproximadamente la mitad del grupo de consumidores en tratamiento y la totalidad del grupo de no consumidores. Sin embargo, en el grupo de consumidores sin tratamiento, hubo personas con las que resultó imposible establecer contacto físico, y se les tuvo que remitir el cuestionario por correo postal, con franqueo pagado, o vía e-mail. La condición de anonimato fue garantizada por escrito.

\section{Instrumentos}

Para explorar los elementos que incluye la TCP, se solicitó a los participantes en el estudio que respondiesen a nueve enunciados directamente relacionados con el consumo de cocaína. En el caso del grupo de sujetos que nunca la habian probado, se les solicitó que respondiesen "como si" lo hubiesen hecho. Se empleó una escala tipo Likert del 1 al 5, en donde 1 significa "Completamente falso para mi", 2 "Bastante falso para mí", 3 "Ni verdadero ni falso para mí, 4 "Bastante verdadero para mí" y 5 "Completamente verdadero".

\footnotetext{
CAIC del Hospital San Juan de Dios, CAID de Alcalá de Henares, CAID de Majadahonda, CAID de Móstoles, CAID de Usera, Centro de Tratamiento EI Olivar, Comunidad Terapéutica Dianova, Comunidad Terapéutica Narconón, Federación de Alcohólicos de la Comunidad de Madrid, Narcóticos Anónimos y Proyecto Hombre.

2 Departamento de Electrónica y Comunicaciones y Departamento de Especialidades Médicas, Psicologia y Pedagogía Aplicadas de la Universidad Europea de Madrid; y, Facultad de Ciencias Políticas y Sociología y Facultad de Ciencias Físicas de la Universidad Complutense de Madrid.
}

Tabla 2. Ítems del Cuestionario TCP divididos por grupos y según coeficiente de confiabilidad (alfa de Cronbach) y porcentaje de varianza total explicada

\begin{tabular}{|c|c|c|c|}
\hline & Grupo A & Grupo B & Grupo $\mathrm{C}$ \\
\hline & alfa & alfa & alfa \\
\hline $\begin{array}{l}\text { Factor I. Actitud (ACT) } \\
\text { 1. Cuando uso cocaina me divierto más. } \\
\text { 2. Consumiendo cocaina se "liga" más. } \\
\text { 3. Consumiendo cocaina se hacen más } \\
\text { amigos. }\end{array}$ & 0,661 & 0,611 & 0,699 \\
\hline $\begin{array}{l}\text { Factor II. Norma subjetiva (NS) } \\
\text { 1. Para mis amistades es importante que } \\
\text { yo también consuma cocaina. } \\
\text { 2. Los amigos me animan a usar cocaina } \\
\text { y estoy dispuesto a hacerles caso. } \\
\text { 3. Mis amigos creen que es bueno con- } \\
\text { sumir cocaina. }\end{array}$ & 0,557 & 0,614 & 0,706 \\
\hline $\begin{array}{l}\text { Factor III. Control Conductual Percibido } \\
\text { (CCP) } \\
\text { 1. Creo que puedo consumir cocaina sin } \\
\text { tener adicción a ella. } \\
\text { 2. Soy capaz de dejar la cocaina cuando } \\
\text { me lo proponga. }\end{array}$ & 0,575 & 0,385 & 0,485 \\
\hline $\begin{array}{l}\text { Factor IV. Intención Conductual (INT) } \\
\text { 1. Estoy dispuesto a seguir consumiendo } \\
\text { cocaina. }\end{array}$ & & & \\
\hline Alfa de Cronbach Total & 0,556 & 0,628 & 0,785 \\
\hline Porcentaje de varianza total explicada & $69,44 \%$ & $57,53 \%$ & $57,75 \%$ \\
\hline
\end{tabular}

Tal y como se observa en la tabla 2, por medio de tres ítems se midió las actitudes de los sujetos ante el consumo de cocaína; con otros tres ítems se evaluó la influencia de la norma subjetiva en el consumo de esta sustancia; se emplearon dos ítems para determinar el control conductual percibido de los sujetos sobre la conducta de consumir cocaína; y con un ítem se precisó la intención que poseian los participantes de consumir esta droga. Estos ítems fueron introducidos por el autor basándose en los estudios arriba mencionados y otros (Pons y Berjano, 1999).

\section{RESULTADOS}

Con el fin de conocer las propiedades estadísticas del cuestionario resultante y la aplicabilidad de los ítems en los tres grupos seleccionados para el estudio, se realizaron las correlaciones de todos los ítems, un análisis factorial y un análisis de fiabilidad, en los tres grupos por separado. 
Tabla 3. Correlaciones

\begin{tabular}{|c|c|c|c|c|c|c|c|c|c|}
\hline \multicolumn{10}{|c|}{ Grupo A } \\
\hline & ACT1 & ACT2 & АCT3 & NS1 & NS2 & NS3 & СCP1 & CCP2 & INT \\
\hline ACT1 & & $0,36^{* *}$ & $0,37^{*}$ & & $0,25^{* *}$ & & & & $0,31^{* *}$ \\
\hline ACT2 & $0,36^{* *}$ & & $0,44^{* *}$ & $0,14^{*}$ & $0,19^{* *}$ & & & & $0,15^{*}$ \\
\hline ACT3 & $0,37^{* *}$ & $0,44^{* *}$ & & $0,17^{*}$ & $0,26^{* *}$ & $0,22^{* *}$ & & & \\
\hline NS1 & & $0,14^{*}$ & $0,17^{*}$ & & $0,38^{* *}$ & $0,36^{* *}$ & & $-0,16^{*}$ & \\
\hline NS2 & $0,25^{* *}$ & $0,19^{* *}$ & $0,26^{*}$ & $0,38^{* *}$ & & $0,15^{*}$ & & $-0,15^{*}$ & \\
\hline NS3 & & & $0,20^{* *}$ & $0,36^{* *}$ & $0,15^{*}$ & & $0,15^{*}$ & & \\
\hline CCP1 & & & & & & $0,15^{*}$ & & $0,40^{* *}$ & $0,32^{* *}$ \\
\hline ССР2 & & & & $-0,16^{*}$ & $-0,15^{*}$ & & $0,40^{* *}$ & & \\
\hline INT & $0,31 *$ & $0,15^{*}$ & & & & & $0,32^{* *}$ & & \\
\hline \multicolumn{10}{|c|}{ Grupo B } \\
\hline ACT1 & & $0,32^{* *}$ & $0,42^{* *}$ & & & & & & $0,30^{* *}$ \\
\hline ACT2 & $0,32^{* *}$ & & $0,27^{*}$ & & & & & & \\
\hline ACT3 & $0,42^{* *}$ & $0,27^{* *}$ & & & $0,32^{* *}$ & $0,23^{*}$ & & & \\
\hline NS1 & & & & & $0,20^{*}$ & $0,59^{* *}$ & & & \\
\hline NS2 & & & $0,32^{* *}$ & $0,20^{*}$ & & $0,20^{*}$ & $0,31 *$ & & $0,19^{*}$ \\
\hline NS3 & & & $0,23^{*}$ & $0,59 *$ & $0,20^{*}$ & & & & \\
\hline CCP1 & & & & & $0,31 *$ & & & $0,25^{* *}$ & $0,36^{* *}$ \\
\hline ССР2 & & & & & & & $0,25^{* *}$ & & $0,20^{*}$ \\
\hline INT & $0,30^{* *}$ & & & & $0,19^{*}$ & & $0,36^{* *}$ & $0,20^{*}$ & \\
\hline \multicolumn{10}{|c|}{ Grupo C } \\
\hline & ACT1 & ACT2 & АСТ3 & NS1 & NS2 & NS3 & CCP1 & ССР2 & INT \\
\hline ACT1 & & $0,21^{*}$ & $0,41^{* *}$ & $0,41^{* *}$ & $0,29^{* *}$ & $0,28^{* *}$ & $0,36^{* *}$ & $0,29^{* *}$ & $0,35^{* *}$ \\
\hline ACT2 & $0,21^{*}$ & & $0,70^{* *}$ & $0,39 *$ & $0,53^{* *}$ & $0,44^{* *}$ & $0,34^{* *}$ & $0,29^{* *}$ & $0,40^{* *}$ \\
\hline ACT3 & $0,41^{* *}$ & $0,70^{* *}$ & & $0,57^{* *}$ & $0,54^{* *}$ & $0,53^{* *}$ & $0,33^{* *}$ & & $0,52^{* *}$ \\
\hline NS1 & $0,41^{* *}$ & $0,39^{* *}$ & $0,57^{* *}$ & & $0,47^{* *}$ & $0,38^{* *}$ & $0,26^{* *}$ & & $0,31^{* *}$ \\
\hline NS2 & $0,29^{* *}$ & $0,53^{* *}$ & $0,54^{* *}$ & $0,47^{* *}$ & & $0,55^{* *}$ & $0,29^{* *}$ & & $0,43^{* *}$ \\
\hline NS3 & $0,28^{* *}$ & $0,44^{* *}$ & $0,53^{* *}$ & $0,38^{* *}$ & $0,55^{* *}$ & & $0,27^{* *}$ & & $0,39^{* *}$ \\
\hline CCP1 & $0,36^{* *}$ & $0,34^{* *}$ & $0,33^{* *}$ & $0,26^{* *}$ & $0,29 * *$ & $0,27^{* *}$ & & $0,35^{* *}$ & $0,36^{* *}$ \\
\hline СCP2 & $0,29 * *$ & $0,29^{* *}$ & & & & & $0,35^{* *}$ & & \\
\hline INT & $0,35^{* *}$ & $0,40^{* *}$ & $0,52^{* *}$ & $0,31 *$ & $0,43^{* *}$ & $0,39^{* *}$ & $0,36 *$ & & \\
\hline \multicolumn{10}{|c|}{$\begin{array}{l}\text { * La correlación es significativa al nivel 0,01 (bilateral). } \\
\text { * La correlación es significante al nivel 0,05 (bilateral). }\end{array}$} \\
\hline
\end{tabular}

De esta manera, tal y como se observa en la tabla 3, los items que conforman cada factor correlacionaron significativamente en cada grupo. En esta dirección, el análisis de componentes principales con rotación Varimax mostró una solidez estructural satisfactoria en todas las submuestras (especialmente la A), agrupando los items en cuatro factores (actitud, norma subjetiva, control conductual percibido e intención), además de un porcentaje de varianza explicada de entre $57,53 \%$ y $69,44 \%$. Finalmente, los distintos análisis de fiabilidad mostraron un alfa de Cronbach suficiente para casi todos los factores y grupos (ver tabla 2).

Por otra parte, para analizar la relación entre los grupos estudiados y las variables de la TCP, teniendo en cuenta el posible efecto de la edad, el género, la edad de inicio del consumo de cocaína y la frecuencia del consumo de la misma, se realizó un análisis de varianza de un factor. El análisis muestra que no existen diferencias significativas entre los grupos estudiados en las variables edad, género y edad de inicio del consumo de cocaína, mientras que sí reveló diferencias significativas en la frecuencia del consumo de cocaína ( $p>$ $0,05)$ (ver tabla 1). Según esto, los resultados obtenidos no se verán influidos por las primeras variables, pero si podrian estar determinados por la mayor frecuencia de consumo de cocaina mostrada por aquellos que actualmente se encuentran en tratamiento.

Tabla 4. Medias de las variables dependientes en los grupos de estudio (rango 1 - 5)

\begin{tabular}{|c|c|c|c|c|c|}
\hline Grupos & & Actitud & Norma Subjetiva & Control Conductual & Intención \\
\hline \multirow{3}{*}{$\begin{array}{l}\text { A. Grupo de } \\
\text { consumidores } \\
\text { sin } \\
\text { tratamiento }\end{array}$} & Media & 2,513a & 1,932a & $2,846 a$ & 3,33a \\
\hline & $\mathrm{N}$ & 192 & 192 & 192 & 192 \\
\hline & Desv. tip. & 0,864 & 0,713 & 0,754 & 1,140 \\
\hline \multirow{3}{*}{$\begin{array}{l}\text { B. Grupo de } \\
\text { consumidores } \\
\text { en } \\
\text { tratamiento }\end{array}$} & Media & $2,154 b$ & 1,945a & $1,604 b$ & $1,26 b$ \\
\hline & $\mathrm{N}$ & 110 & 110 & 110 & 110 \\
\hline & Desv. tip. & 1,035 & 0,968 & 0,851 & 0,725 \\
\hline \multirow{3}{*}{$\begin{array}{l}\text { C. Grupo } \\
\text { Control }\end{array}$} & Media & $1,406 c$ & $1,206 b$ & $1,38 b$ & $1,35 b$ \\
\hline & N & 100 & 100 & 100 & 100 \\
\hline & Desv. tip. & 0,648 & 0,425 & 0,569 & 0,730 \\
\hline \multicolumn{6}{|c|}{$\begin{array}{l}\text { Nota: Se añaden por orden las letras }(a>b>c) \text { cuando en las medias obtenidas } p<0,05 \text {, según la prueba } \\
\text { post hoc Bonferroni de comparaciones múltiples. }\end{array}$} \\
\hline
\end{tabular}

Seguidamente se analizaron los efectos del grupo de estudio sobre las variables de la TCP. Para esto se obtuvieron las medias de las variables estudiadas sumando los ítems de cada factor y dividiéndolos entre el número de ítems que lo componian. Como puede observarse en la tabla 4, el grupo de consumidores sin tratamiento puntuó significativamente más alto en las variables actitud $(p<0,01)$, control conductual percibido $(p<0,001)$ e intención conductual $(p<0,001)$. Sin embargo, aunque también puntuó por encina del grupo que nunca había consumido en la variable norma subjetiva $(p<0,001)$, se mantuvo en el mismo nivel que el grupo de consumidores en tratamiento.

El grupo de consumidores en tratamiento puntuó más alto que aquellos que nunca habian consumido cocaína en la actitud hacia este sustancia $(p<0,001)$ y en la norma subjetiva $(p<0,001)$. No obstante, mantuvo el mismo bajo nivel que estos últimos en las variables control conductual percibido e intención de consumo.

Finalmente, el grupo de sujetos que nunca habian consumido cocaína fue el que puntuó más bajo en todas las variables menos en intención conductual, que se localizó prácticamente al mismo bajo nivel que aquellos que se encontraban en tratamiento.

\section{DISCUSIÓN}

En primer lugar, es de reconocer que el conjunto de ítems empleados para medir las variables estudiadas, aunque han demostrado unas propiedades estadísticas aceptables, están lejos de poder ser considerados como una escala definitiva para el abordaje de los elementos que propone la TCP (ejem. Olivar y Carrero, 2007). En este sentido, resulta necesario subrayar la necesidad de contrastar los resultados aquí obtenidos y verificar, por otros medios, si los elementos que se han mostrado tan significativos en este estudio para describir a los grupos analizados, continúan comportándose de igual manera. 
En cuanto a los resultados obtenidos, resulta necesario tomar en cuenta, aunque sea de forma hipotética, las implicaciones que éstos pudieran significar. Así, encontramos que aquellas personas que consumen cocaina mostraron la actitud más favorable al consumo de esta sustancia, aunque la puntuación media que estos sujetos le otorgaron, podría suponer que no es la variable más importante de las que la TCP comprende $(A=2,513$; rango 1-5). Además, quienes están en tratamiento por consumo de cocaína, mostraron una actitud no completamente desfavorable al consumo de la misma $(B=2,154$; rango $1-5)$, la cual era significativamente superior a la de aquellos que nunca habian consumido esta sustancia $(C=1,406 ; p<0,001)$. Es posible que esto se deba a que, conjuntamente con la capacidad de la cocaína para actuar como reforzador negativo (evitando al ser consumida el malestar que su ausencia en el organismo puede implicar), muchas de las personas en tratamiento mantenían la convicción de que consumiendo cocaína se podian obtener beneficios adicionales (ligar, divertirse, hacer más amigos o mejorar sus relaciones sexuales) (Calafat, Juan, Becoña y Mantecón, 2008).

De forma similar a lo anterior, encontramos una mayor influencia del grupo de amigos en quienes consumen cocaína que en quienes nunca lo han hecho $(A=1,932 ; C=1,206$; $p<0,001)$. Sin embargo, los sujetos consumidores tampoco reconocieron una influencia activa de sus amistades para consumir cocaína (rango 1-5), recordando el debate sostenido por Urberg, Luo, Pilgrim y Degirmencioglu (2003), en el cual se discute el grado en el que un sujeto es influido para consumir o éste elige a sus amigos para consumir ${ }^{3}$. También se pudo encontrar que, aunque actualmente la norma subjetiva tampoco parecía ejercer una influencia importante entre el grupo de sujetos en tratamiento (Media=1,945; rango 1-5), ésta era significativamente mayor que entre aquellas personas que nunca habian consumido cocaina (Media=1,206; $p<0,001)$. Asi, es probable que algunos sujetos en tratamiento aun tuviesen amistades consumidoras que les animasen a reincidir.

Por otra parte, hallamos como factor muy significativo, el hecho de que aquellos sujetos que consumen cocaina consideraron que pueden controlar esta conducta, mientras que quienes nunca la han consumido o están en tratamiento, creen que existe la posibilidad de que el uso de esta sustancia les lleve a la adicción $(A=2,846 ; B=1,604 ; p<0,001$ y $A=2,846 ; C=1,38 ; p<0,001)$. Esto confirmaría lo expuesto por otros autores (Megías, Comas, Elzo, Navarro y Romani, 2001; Megías, 2005), acerca de que la percepción de la cocaína, como una droga de la cual puede hacerse uso sin complicaciones, es uno de los principales factores de riesgo para el consumo de la misma. Además, no encontramos diferencias significativas en la variable control conductual percibido entre los grupos B y C. Esto nos podría indicar que, tanto aquellas personas que están en tratamiento por adic-

\footnotetext{
Por otra parte, es posible que también hubiese sido más productivo introducir el término de "compañías" en lugar del de "amigos", ya que algunos de los encuestados reconocieron que aunque ciertamente consumen con otras personas, sus "verdaderos amigos" son ajenos a estas prácticas.
}

ción a la cocaína, como las que nunca la han consumido, no consideran tener la capacidad para hacer uso de esta droga de una manera controlada y perciben el riesgo adictivo que la cocaína encierra.

Por último, quienes consumen cocaína mostraron más intención de seguir consumiéndola que quienes nunca la han probado o están en tratamiento $(A=3,33 ; B=1,26 ; p<0,001$; y $A=3,33 ; C=1,35 ; p<0,001)$, siendo de resaltar que los sujetos de los grupos $B$ y $C$ mostraron la misma nula intención de consumir esta sustancia en el futuro (rango 1-5). Por otra parte, el hecho de que los consumidores de cocaina no puntuasen más elevado en este ítem $(3,33 / 5)$, podría también deberse a la carga estigmatizante que esta conducta impli$c a$, y la dificultad de reconocer públicamente sus planes de seguir realizándola (Link, Struening, Rahav, Phelan y Nuttbrock, 1997; Saiz, 2008a).

Dentro de un nivel teórico, el estudio aquí expuesto muestra que la TCP puede ser empleada para la medición y explicación de la conducta de uso y abuso de drogas. No obstante, debemos reconocer que, como lo describen otros autores (Bagozzi y Kimmel, 1995; Leone, Perugini y Ercoloni, 1999; Ros, 2001), es posible que esta teoría tenga la limitación de no incluir un importante número de elementos que también ha demostrado su eficacia en este ámbito (Becoña, 1999, 2002; Saiz, 2008b). En este sentido, deberemos ser cautos al interpretar los resultados obtenidos y tomar en cuenta que la magnitud del problema que nos ocupa exige su estudio desde posiciones interdisciplinarias complementarias (Liska, 1984; Kuther, 2002).

De esta manera, partimos del punto de que las variables que explora la TCP son fundamentalmente de corte sociocognitivo, y resulta necesaria la inclusión de elementos que comprendan también una dimensión emocional, como los rasgos de personalidad (Pedrero, 2007; Teichman, Barnea y Ravav, 1989), sociológica (Ben-Yehuda, 1984; Saiz, 2007), biológica (Pineda y Torrecilla, 1999; Wise, 1999), etc. para alcanzar una descripción lo más acertada posible.

Por otra parte, tomando en cuenta que "la intervención especifica en materia de drogas constituye una de las más acuciantes demandas sociales e institucionales" (Moral, Ovejero, Sirvent y Rodríguez, 2005), y partiendo de la exigencia de realizar programas preventivos basados en la evidencia científica (AA., 2002; Hawkins, Catalano y Arthur, 2002), con esta investigación se ha tratado de aportar una serie de conocimientos que añadan datos relevantes al problema del consumo actual de cocaína. Así, independientemente de los ámbitos de aplicación de los programas preventivos (Espada, García, Méndez y Lloret, 2005; Martín y Moncada, 2003; Moral, Ovejero, Sirvent y Rodríguez, 2005), los resultados aquí obtenidos nos obligan a enfatizar la pertinencia de abordar las actitudes, la norma subjetiva y el control conductual percibido dentro de las distintas campañas de prevención. Además, la variable control conductual percibido indicaba que uno de los determinantes más directos a tomar en cuenta para entender el consumo actual de cocaína es el hecho de que los sujetos creen que pueden controlar su consumo, no mostrando preocupación por su capacidad adictiva. A falta de estudios que expongan la eficacia de las distintas 
campañas preventivas, esto apoyaria la campaña número 31 puesta en marcha por la Fundación de Ayuda contra la Drogadicción "Cambia tu percepción-Piensa" (enero, 2007).

Posiblemente, la solución a este problema pase, como señalan Martín y Moncada (2003), por "plantear un auténtico debate social sobre cómo educar para el ocio, corresponsabilizando a la industria, implicando a las instituciones públicas y sensibilizando a las familias y a la sociedad para desarrollar un ocio saludable y positivo". Así, parece prioritario basar las distintas campañas en ofrecer una imagen real de la cocaína y de la influencia del grupo de iguales en el curso de su consumo, pero especialmente, relacionar el uso que actualmente parece dársele a esta sustancia en distintos ambientes de ocio con sus posibles consecuencias.

Para finalizar, sólo resta matizar el carácter descriptivo de este trabajo y subrayar la necesidad de concentrar esfuerzos en estudios longitudinales, que pongan a prueba las distintas opciones preventivas y faciliten una perspectiva de intervención eficaz, adaptada a las características de los consumidores actuales de cocaina y dirigida a evitar los problemas derivados del abuso y dependencia de esta sustancia.

\section{AGRADECIMIENTOS}

Este estudio fue realizado gracias a la contribución económica de la Fundación Ramón Areces. Además, debo expresar mi gratitud a todas las personas que, en diferentes momentos, apoyaron el trabajo de campo que el mismo implica.

\section{REFERENCIAS}

AA. (2002). Catálogo de Programas de Prevención del abuso de drogas. Madrid: Consejería de Sanidad y Consumo, Agencia Antidroga Comunidad de Madrid.

Ajzen, I. (1991). The Theory of Planned Behavior. Organizational Behavior and Human Decision Processes, 50, 179-211.

Ajzen, I. (1988). Attitudes, personality and behavior. Chicago: Dorsey Press.

Ajzen, I. y Fishbein, M. (1980). Understanding attitudes and predicting social behavior. Nueva York: Prentice Hall.

Bagozzi, R. y Kimmel, S. (1995). A comparison of leading theories for the prediction of goal-directed behaviours. British Journal of Social Psychology, 34, 437-461.

Becoña, E. (1999). Bases Teóricas que sustentan los programas de prevención de drogas. Madrid: PNSD.

Becoña, E. (2002). Bases cientificas de la prevención de las drogodependencias. Madrid: PNSD.

Ben-Yehuda, N. (1984). A clinical sociology approach to treatment of deviants: the case of drug addicts. Drug and Alcohol Dependence, 13, 267-282.

Calafat, A., Juan, M., Becoña, E., Fernández, C., Gil, E., Palmer, A., et al. (2000). Salir de marcha y consumo de drogas. Madrid: Plan Nacional sobre Drogas, Caja Madrid.
Calafat, A., Juan, M., Becoña, E., y Mantecón, A. (2008). Qué drogas se prefieren para las relaciones sexuales en contextos recreativos. Adicciones, 20, 37-48.

Conner, M. y McMillan, B. (1999). Interaction effects in the theory of planned behavior: Studying cannabis use. British Journal of Social Psychology, 38, 195-222.

Conner, M., Sandberg, T., McMillan, B. y Higgins, A. (2006). Role of anticipated regret, intentions and intention stability in adolescent smoking initiation. British Journal of Health Psychology, 11, 85-101.

Côté, F., Godin, G. y Gagné, C. (2004). Identification of factors promoting abstinence from smoking in a cohort of elementary schoolchildren. Preventive Medicine, 39, 695-703.

Espada, J., Garcia, J., Méndez, F. y Lloret, D. (2005). Estrategias para la prevención escolar del consumo de drogas de fin de semana. En J. García y C. López (Coord.). Manual de estudios sobre cocaina y drogas de sintesis. Madrid: Edaf.

Estrategia Nacional Sobre Drogas 2000-2008. (1999). Madrid: Ministerio del Interior-Delegación del Gobierno para el Plan Nacional sobre Drogas.

Hawkins, J., Catalano, R. y Arthur, M. (2002). Promoting science-based prevention in communities. Addictive Behaviors, 27, 951-976.

Hulten, R., Bakker, A. Lodder, A., Teeuw, K., Bakker, A. y Leufkens, $H$. (2003). The impact of attitudes and beliefs on length of benzodiazepine use: a study among inexperienced and experienced benzodiazepine users. Social Science and Medicine, $56,1345-1354$.

Humphrey, R., O'Malley, P., Johnston, L. y Bachman, J. (1988). Bases of power, facilitation effects, and attitudes and behavior: direct, indirect, and interactive determinants of drug use. Social Psychology Quarterly, 51, 329-345.

Kuther, T. (2002). Rational decision perspectives on alcohol consumption by youth: Revising the theory of planned behavior. Addictive Behaviors, 27, 35-47.

Leone, L., Perugini, M. y Ercolani, A. (1999). A comparison of three models of attitude-behavior relationships in the study behavior domain. European Journal of Social Psychology, 29, 161-189.

Levy, S. y Pierce, P. (1989). Predicting intention to use cocaine in teenagers in Sydney, Australia. Addictive Behaviors, 14, 105-111.

Link, B., Struening, E., Rahav, M., Phelan, J. y Nuttbrock, L. (1997). On stigma and its consequences: evidence from a longitudinal study of men dual diagnoses of mental illness and substance abuse. Journal of Health and Social Behavior, 38, 177-190.

Liska, A. (1984). A critical examination of the causal structure of the Fishbein/Ajzen Attitude-Behavior Model. Social Psychology Quarterly, 47, 61-74.

Martín, E. y Moncada, S. (2003). Programas de prevención de ocio alternativo. Adicciones, 15, 327-346.

McMillan, B. y Conner, M. (2003). Using the theory of planned behaviour to understand alcohol and tobacco use in students. Psychology, Health \& Medicine, 8, 317-328.

Megias, E., Comas, D., Elzo, J., Navarro, J. y Romani, O. (2001). La percepción social de los problemas de drogas en España. Madrid: FAD.

Megías, E. (2005). La percepción social de los problemas de drogas en España, 2004. Madrid: FAD y Caja Madrid.

Moral, M., Ovejero, A., Sirvent, C. y Rodríguez, F. (2005). Prevención e intervención psicosocial sobre el consumo juvenil de sustancias psicoactivas: Comparación de la eficacia preventiva de cuatro programas. Intervención Psicosocial, 14, 198-208. 
Navarro, J. (2000). Factores de riesgo y protección de carácter social relacionados con el consumo de drogas. Madrid: Ayuntamiento de Madrid.

Norman, P. y Conner, M. (2006). The theory of planned behaviour and binge drinking: Assessing the moderating role of past behaviour within the theory of planned behaviour. British Journal of Health Psychology, 11, 55-70.

O'Callaghan, F. y Hannon, T. (2003). Normalization of marijuana use: its effects on adolescents' intentions to use marijuana. Substance Use and Misuse, 38, 185-199.

OED. (2005). Informe 2004. Madrid: Ministerio del Interior.

Olivar, Á. y Carrero, V. (2007). Análisis de la intención conductual de consumir cannabis en adolescentes: desarrollo de un instrumento de medida basado en la teoría de la conducta planificada. Trastornos Adictivos, 9, 184-205.

Pineda, J. y Torrecilla, M. (1999). Mecanismos neurobiológicos de la adicción a drogas. Trastornos Adictivos, 1, 13-21.

Pedrero, J. (2003). Los trastornos de la personalidad en drogodependientes desde la perspectiva de los cinco grandes factores. Adicciones, 15, 203-220.

Pedrero, J. (2007). El modelo de los cinco factores y la estructura dimensional de los trastornos de la personalidad: estudio de una muestra de adictos a sustancias con el BFO y el MCMI-II. Trastornos Adictivos, 9, 116-125.

Pons, J. y Berjano, E. (1999). El consumo abusivo de alcohol en la adolescencia: un modelo explicativo desde la Psicología Social. Madrid: PNSD.

Ros, M. (2001). Valores, actitudes y comportamiento: una nueva visita a un tema clásico. En M. Ros y V. V. Gouveia (Coords.). Psicología social de los valores humanos. Madrid: Biblioteca Nueva.

Sáiz, P. A., González, M. P., Paredes, B., Martínez, S. y Delgado, J. M. (2001). Personalidad y uso-abuso de cocaina. Adicciones, 13, $47-60$.
Saiz, J. (2007). El abuso de cocaina, ¿Problema de oferta o de demanda social?: un estudio transcultural y correlacional que compara variables macrosociales, económicas y culturales. Adicciones, 19, $35-44$.

Saiz, J. (2008a). El estigma de la cocaina. Revista Española de Sociología, 10, 97-114.

Saiz, J. (2008b). Psicología Social de la Salud sobre el consumo adictivo de Cocaina: Un modelo psicosocial sintético. Intervención Psicosocial, 17, 61-74.

Schifter, D. y Ajzen, I. (1985). Intention, Perceived Control, and Weight Loss: An Application of the Theory of Planned Behavior. Journal of Personality and Social Behavior, 49, 843-851.

Synnøve, I. y Rise, J. (2006). Predicting smoking reduction among adolescents using an extended version of the theory of planned behaviour. Psychology and Health, 21,717-738.

Teichman, M., Barnea, Z. y Ravav, G. (1989). Personality and substance use among adolescents: a longitudinal study. British Journal of Addiction, 84, 181-190.

Trujillo, A., Forns, M. y Pérez, A. (2007). Uso de sustancias y percepción de riesgo: Estudio comparativo entre jóvenes de Bogotá y Barcelona. Adicciones, 19, 179-190.

Umeh, K. y Patel, R. (2004). Theory of planned behaviour and ecstasy use: An analysis of moderator-interactions. British Journal of Health Psychology, 9, 25-38.

UNODC (2007). World Drug Report 2007. Recuperado el 27 de junio de 2007 de: www.unodc.org.

Urberg, K., Luo, O., Pilgrim, C. y Degirmencioglu, S. (2003). A twostage model of peer influence in adolescent substance use: individual and relationship-specific differences in susceptibility to influence. Addictive Behaviors, 28, 1243-1256.

Wise, R. (1999). Neurobiología de la adicción. Revista de Toxicomanías, 18, 15-25. 